\title{
Current Concepts in the Management of Muscle Invasive Bladder Cancer
}

\author{
Suprita R. Krishna ${ }^{1} \cdot$ Badrinath R. Konety ${ }^{1}$
}

Received: 17 October 2016/Accepted: 24 October 2016/Published online: 15 December 2016

(C) Indian Association of Surgical Oncology 2016

\begin{abstract}
Bladder cancer is the ninth most common cancer in the world. Twenty to twenty-five percent of all newly diagnosed bladder cancers are muscle invasive in nature, and further, 20-25\% of patients who are diagnosed with high-risk nonmuscle invasive disease will eventually progress to muscle invasive disease in due course of time irrespective of adjuvant intravesical therapies. Availability of newer imaging modalities improves appropriate identification of patients with muscle invasive disease. Radical cystectomy remains the mainstay of treatment for management of muscle invasive disease. Availability of neoadjuvant chemotherapy has improved overall survival. Risk stratification systems are now in consideration to identify patients who benefit maximally from neoadjuvant chemotherapy. Urinary diversion is a major cause of morbidity in these patients, and several strategies are being employed to reduce morbidity. In this article, we review available literature on various aspects of management of muscle invasive disease.
\end{abstract}

Keywords Bladder cancer - Muscle invasive bladder cancer . Blue light cystoscopy · PET CT · Neoadjuvant chemotherapy for bladder cancer $\cdot$ Cystectomy

\section{Introduction}

Bladder cancer is the ninth most common cancer in the world, with 430,000 new cases diagnosed in 2012 [1]. Bladder cancer is three times more common in men than women, with the

Badrinath R. Konety

brkonety@umn.edu

1 Department of Urology, University of Minnesota, Minneapolis, MN 55455, USA median age at diagnosis being 73 [2]. According to SEER data, it is the fifth most common cancer in the USA. There were an estimated 75,000 new cases (56,390 men and 18,300 women) and 15,580 (11,170 men and 4410 women) deaths in the year 2014 [3].

Histologically, over $90 \%$ of bladder tumors are transitional cell carcinomas. The other subtypes, such as squamous cell and adenocarcinoma, are uncommon and account for 5 and $<2 \%$, respectively. Bladder cancer is a heterogeneous disease with a variable natural progression. Urothelial carcinomas can have a component of "variant histology" such as squamous differentiation, micropapillary, or adenocarcinoma. Micropapillary variant of urothelial carcinoma is associated with worse prognosis than pure urothelial carcinoma. Around $70 \%$ of the patients present with tumors that are superficial and have a tendency to recur. The remaining $30 \%$ present with muscle invasive tumors [4].

\section{Pathology}

Low-grade tumors represent $50-60 \%$ of the cases. Majority of the tumors are stage Ta, i.e., confined to the urothelium. These tumors tend to recur and generally require repeated transurethral resection (TURBT) and surveillance cystoscopies [5].

High-grade (HG) lesions account for $20-25 \%$ of all new cases. These lesions can either be sessile or papillary lesions. These tumors have a tendency to invade into the bladder wall if left untreated, especially in the presence of carcinoma in situ (CIS). Despite treatment in the form TURBT and chemotherapy, more than $20 \%$ of the patients with high-grade lesions progress to muscle invasive bladder cancer (MIBC) [6, 7]. This has led to a lot of controversy regarding aggressive treatment in the form of early radical cystectomy vs. TURBT along with intravesical therapy, particularly in patients with HG 
disease that is recurrent or invades the lamina propria but not the muscle. Though cystectomy provides the best chance for cure, it decreases the overall quality of life. Among non muscle invasive bladder cancer (NMIBC), non-invasive Ta lesions tend to be less aggressive than $\mathrm{T} 1$ lesions that invade the lamina propria. T1 lesions generally tend to be high grade. One problem with HG T1 lesions is the high potential for under staging. This is often due to inadequate sampling of the muscularis propria or even complete absence of muscle in the initial resection specimen. The prevalence of under staging can be as high as $40 \%$ [8]. Therefore, pathological HG T1 lesions warrant resampling with a repeat TURBT performed within 4-6 weeks of the initial resection, even if there was a muscle in the initial specimen, to verify the absence of muscle invasion and for more appropriate stage assignment.

MIBC accounts for 20-25\% of all newly diagnosed cases. In these cases, the tumor has invaded the muscularis propria. Despite undergoing radical cystectomy, the 5-year cancer-specific survival is estimated to be $60 \%$, with approximately $50 \%$ of the patients succumbing to metastatic disease $[9,10]$. Invasion of the detrusor muscle and development of hydronephrosis are two important events in disease progression associated with lymph node (LN) positive disease and poor prognosis. [5, 11] In a study on 753 patients, 244 (32\%) had hydronephrosis. This study concluded that the side of hydronephrosis predicted the side of lymph node involvement in $84 \%$ of the patients [11].

\section{Presentation and Diagnosis}

The most frequently encountered presenting symptom is painless gross hematuria [2, 12]. Occasionally, in the setting of CIS, the patient may present with voiding symptoms such as urinary frequency, dysuria, urgency, or asymptomatic hematuria [4]. Asymptomatic microscopic hematuria is defined as the presence of three or more red blood cells (RBC) per high-power field (hpf). In the setting of gross hematuria, the decision to evaluate further is the norm. In subjects with asymptomatic hematuria, the incidence of bladder cancer can be as high as $20 \%$. Bladder cancer has been detected in $31.5 \%$ of patients with gross hematuria and $15.7 \%$ with microscopic hematuria [13]. This reiterates the importance of evaluating hematuria.

Voided or catheterized urinary specimen should be sent for microscopic evaluation and culture to rule out foci of infection. A urine dipstick will not suffice, as it cannot differentiate between fresh RBC, myoglobin, and lysed RBC seen in hemoglobinopathies [14]. In low-risk patients, urine cytology may suffice for initial screening. Though urine cytology carries $40-76 \%$ sensitivity in detecting bladder cancer, it depends on factors such as the grade and stage of the tumor [15]. Cytology is not very sensitive in detecting low-grade tumors, and this can be disadvantageous because $50-60 \%$ of newly diagnosed cases of bladder cancer are low-grade tumors [5, 13].

There are currently at least four approved and widely available urine-based markers (NMP22 BladderChek, BTA, Urovysion FISH and ImmunoCyt), which are reported to have better sensitivity than cytology for detecting low-grade bladder tumors [16-19]. Two of these markers (NMP22 BladderChek and BTA) are cell-based markers and are more prone to be affected by hematuria and inflammation. Urovysion FISH and ImmunoCyt are cell-based markers and tend to have higher sensitivity than protein-based markers. However, all the markers have shown lower specificity than voided urine cytology.

\section{Cystoscopy}

White light cystoscopy (WLC) is the primary detection method for bladder cancer [20] and is routinely performed to evaluate gross hematuria. Current AUA guidelines suggest that asymptomatic microhematuria in patients above 35 years of age and patients less than 35 years with risk factors (current or past tobacco use, irritative voiding symptoms, chemical exposure, pelvic irradiation) should be evaluated with cystoscopy [21].

Newer optical agents have been introduced in the recent years. Fluorescent cystoscopy (FC) has particularly shown positive outcomes when used as an adjunct to WLC. For FC, hexaminolevulinate (HAL) dye is instilled intravesically, and it fluoresces when visualized under blue light (refer to Fig. 1a, b). Several trials have evaluated the use of HALBLC in the routine evaluation of bladder cancer. The sensitivity of FC and WLC to detect cancer is 87 and $83 \%$, and the specificity is 92 and $68 \%$, respectively [22]. FC offers a means for improved tumor detection and more complete resection of tumors to prevent recurrence. This is mainly useful for cases of CIS and non-muscle invasive bladder cancer [23].

\section{Improved Diagnostic Techniques}

Optical coherence tomography (OCT) is a new imaging technology, which uses near infrared and the back scattering pattern of various tissues to differentiate cells from stroma and assesses the depth of invasion. OCT provides high resolution (20$30 \mu \mathrm{m}$ ), cross sectional imaging of tissue. Using OCT, one can visualize the mucosa, lamina propria, and the superficial muscularis as layers and identify structural changes in them. It is able to distinguish benign from malignant disease with positive predictive value of $89 \%$ and negative predictive value of $100 \%$. With a $100 \%$ sensitivity and $92 \%$ specificity when it comes to defining MIBC, OCT is valuable to prevent overlooking muscle invasion and to ensure adequate depth of resection of the tumor [24].

Narrow-band imaging (NBI) uses modified optical filters which split white light into two bands, which are absorbed by hemoglobin. Bladder tumors are highly vascular, and the 

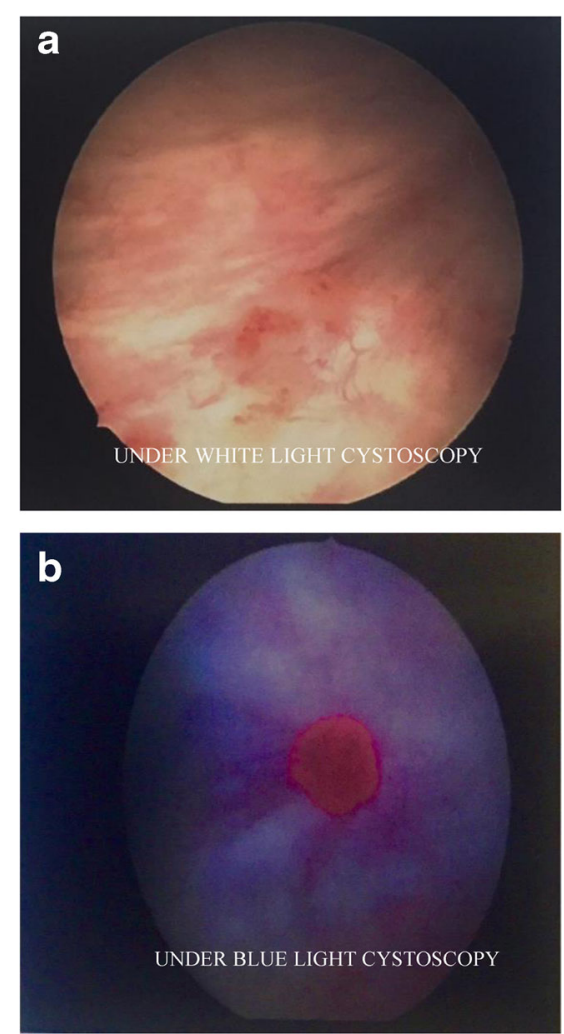

Fig. 1 Hexaminolevulinate (HAL) dye under white light cystoscopy and under blue light cystoscopy

visibility of the capillaries on the surface and the submucosa is increased. This is a novel method to distinguish between normal and abnormal mucosa. The advantage of NBI is that it can easily be coupled with a standard endoscopic platform; it requires no other markers or dyes and can be performed in real time [25]. Under NBI, the vasculature appears dark green to black against a pale white mucosa, thus enhancing the contrast between tumor and normal epithelium. NBI has been shown to improve detection of recurrent flat and papillary superficial bladder tumors and margins are better defined. It has a false positive rate of $13 \%$, raising concern about unnecessary bladder biopsies and the potential morbidity associated with it [26]. Nonetheless, NBI has excellent tumor detection rates and can therefore be an aide to traditional WLC.

\section{Imaging}

\section{$C T$}

CT urography is the primary imaging study performed in bladder cancer, especially in cases where there is a definite reason to suspect a high-grade lesion (high-risk patients, macroscopic hematuria) [27]. CT urography is particularly helpful beyond stage T3b, i.e., involvement of the perivesical fat and surrounding organs and in assessing the ureters and renal pelvis [28]. Sensitivity of CT imaging for diagnosing perivesical invasion is less compared to MRI ( 89 vs. $100 \%$ ), but its specificity is greater (95 vs. $73 \%$ ) [29].

MRI

MRI is now emerging as an important method of staging bladder cancer. MRI has $100 \%$ sensitivity for detecting perivesical invasion. Since MRI is very sensitive, even mild inflammation around the cancerous region can mimic perivesical invasion. This results in over staging of these tumors [28].

\section{Lymph Node Imaging}

As a general rule, pelvic nodes more than $10 \mathrm{~mm}$ in short axis dimension are considered abnormal. When pelvic lymphadenopathy is identified, the retroperitoneum should be screened for paraaortic lymphadenopathy. The presence of paraaortic lymph nodes makes the tumor unresectable [30].

Studies have shown that both CT and MRI are comparable in detecting lymph node metastasis, with a low sensitivity between 48 and $87 \%$. The specificity is low as well, since lymph nodes also enlarge due to benign pathology [28].

\section{PET/CT Scan}

Positron emitted tomography/CT (PET/CT) using F-18 fluorodeoxyglucose (FDG) has become an important imaging modality for staging cancer. With CT and MRI having false negative rates up to $40 \%$, PET/CT offers a more accurate and non-invasive preoperative staging method [31-33]. CT and MRI use lymph node size as the criterion for diagnosis, which leads to overlooking micrometastasis in normal-sized lymph nodes. Malignant cells are characterized by increased glucose metabolism. Therefore, malignant cells can be visualized as increased uptake of FDG on PET/CT [34, 35].

The drawbacks of FDG are its short half life (110 $\mathrm{min})$ and are excreted by the kidneys, accumulating in the urinary tract, making it difficult to visualize the urinary system in depth [36]. Its current applications in bladder cancer work-up include evaluation of lymph node involvement and distant metastasis. With a pooled sensitivity of $82 \%$ and pooled specificity of $89 \%$ based on a meta-analysis of six studies, PET/CT provides better insight compared to CT/MRI. PET/CT detected more malignant disease in $40 \%$ of the patients compared to $\mathrm{CT}$ and MRI, and clinicians changed the plan of management in $68 \%$ of the patients based on results from PET/CT [37].

Bone scan has $38 \%$ sensitivity for detecting cancer, but it has low specificity since reactive changes can also cause isotope uptake. The negative predictive value is $92 \%$. Unless the patient has symptoms of bone pain or an elevated alkaline phosphatase level, bone scan can be avoided in most cases. If a PET/CT is obtained, it will provide information about bone involvement as well. 


\section{Management of MIBC}

\section{Neoadjuvant Chemotherapy}

The use of neoadjuvant chemotherapy (NCT) has been steadily increasing over the past few years. In 2006, $10.2 \%$ of the patients undergoing cystectomy received $\mathrm{NCT}$, and the rate has steadily increased to $20.9 \%$ in 2010 [38]. NCT is reserved for patients with stage T2T4a operable tumors and is aimed at treating micrometastasis concurrently. In addition to being better tolerated prior to surgery, preoperative chemotherapy has the added advantage of being able to downstage the tumor, potentially making surgery easier. [39, 40] It also offers a method to survey the in vivo efficacy of the chemotherapy, which is an important prognostic indicator for cancer-free survival [41].

The largest study on NCT was conducted by the European Organization for Research and Treatment of Cancer (EORTC) and the Medical Research Council (MRC). They recruited 976 patients with $\mathrm{T} 2$ grade $3, \mathrm{~T} 3 / \mathrm{T} 4, \mathrm{~N} 0$, and M0 bladder cancer to receive 3 cycles of cisplatin, methotrexate, and vinblastine (CMV) or no therapy prior to cystectomy or radiotherapy. Median survival in the chemotherapy group was 44 months, compared to 36.5 months in patients who received no chemotherapy. $32.5 \%$ of cystectomy samples contained no tumor after chemotherapy [42].

Currently, the two main NCT combinations used are methotrexate, vinblastine, adriamycin, and cisplatin (MVAC) and cisplatin with gemcitabine (GC). The Southwest Oncology Group conducted a study to test MVAC. Three hundred seven patients were enrolled into the study, with MIBC (stage T2 to T4a). The patients were stratified according to age $(<65$ and $>65$ ) and depth of muscle invasion (superficial vs. more extensive) and were assigned to receive 3 cycles of MVAC followed by cystectomy or cystectomy alone. One hundred fifty-four patients received cystectomy alone, and 153 received combination therapy. Patients treated with MVAC did better, with median survival of 77 vs. 46 months in patients who received cystectomy alone. More patients in the combination therapy group had no residual disease compared to the cystectomy group ( 38 vs. $15 \%$ ). One third of the patients who received MVAC developed severe gastrointestinal and hematological toxicity, but all of them recovered. It did not increase the patient's risk for complications after surgery nor did it result in them being unfit for cystectomy. The estimated risk of death was reduced by $33 \%$ in patients assigned to receive MVAC and cystectomy compared to cystectomy alone [43].

Currently, GC is the most widely utilized NCT regimen. There is a lack of level 1 evidence supporting the use of GC, and the initial data supporting its use is based on a phase 3 clinical study on MVAC vs. GC involving 405 patients with metastatic disease. The study concluded that the overall progression-free survival was similar in both MVAC and GC. The GC regimen was associated with considerably lesser toxicity compared to MVAC [44].

The limitations of neoadjuvant chemotherapy include (1) potential delay between diagnosis and definitive management (cystectomy) [45], (2) depending on clinical rather than pathological staging to administer NCT, and (3) dearth of prognostic biomarkers to determine response to NCT [41].

A study by Dinney et al. suggested a risk-stratified approach to help decide which patients with MIBC received NCT. They categorized patients as high risk based on the presence of features such as: (1) hydroureteronephrosis, (2) cT3b and cT4a disease, (3) lymphovascular invasion, and (4) variant histology on TURBT. The study concluded that patients with high-risk features have decreased overall 5year survival, disease-specific survival, and progression-free survival compared to the low-risk group. This distinction between high-risk and low-risk patients helps identify the patients who would most benefit from NCT prior to cystectomy and also spares low-risk patients the morbidity and systemic side effects of NCT [46].

\section{Radical Cystectomy}

The standard form of therapy for patients with MIBC is a cystoprostatectomy for males and an anterior pelvic exenteration (bladder, urethra, uterus, ventral vaginal wall) for females. For less extensive disease, the surgery can be restricted to cystectomy only in females, particularly in younger women who desire preservation of reproductive function [4]. Pelvic lymph node dissection is performed concurrently. After a radical cystectomy, urinary diversion can be either non-continent or continent, and both constructed using a segment of bowel. The cystectomy can be performed using open, laparoscopic, or robotic technique, each presenting its own advantages and shortcomings.

The most commonly used approach for cystectomy is open radical cystectomy (ORC) with extended pelvic lymph node dissection [47]. ORC is associated with high morbidity in the form of increased blood loss, longer hospital stay, and longer recovery time. The recurrence-free survival rate for MIBC at 5 years after cystectomy is around $70 \%$. Recurrence-free survival drops to $50-60 \%$ with extravesical spread and down to $35 \%$ when lymph nodes are involved [48].

Both laparoscopic radical cystectomy (LRC) and robot-assisted radical cystectomy (RARC) have longer operating time (RARC more than LRC), but studies show both produce better surgical outcomes with reduced blood loss and shorter length of stay in the hospital [49]. Another important point to factor in is the challenging and long learning curve associated with both LRC and RARC. The rate of positive surgical margins and estimated blood loss has been shown to decrease and 
reach a plateau, with increasing number of cases performed by the surgeon [50].

Pelvic lymph node dissection (PLND) is of utmost importance to determine the prognosis of individuals and to identify the patients who will benefit from adjuvant chemotherapy, as node positive disease is associated with high risk for relapse [51]. Lymph node dissection can either be standard or extended. Standard pelvic lymph node dissection includes the distal common iliac, external iliac, hypogastric, and obturator nodes [52]. The extended pelvic lymph node dissection differs from the standard PLND in that, its upper limit is the inferior mesenteric artery [53]. Currently, it is generally reported that 10 15 lymph nodes have to be removed for it to be considered an adequate lymph node dissection.

\section{Radical TURBT}

Radical TURBT can be considered for patients who have a single tumor, that is, $<3 \mathrm{~cm}$, no CIS present, and there is no hydronephrosis. Such tumors can be resected completely and is the approach used in patients with poor performance status. In one study of 432 patients with MIBC, 99 patients were treated with TURBT alone. The 10-year disease-specific survival was $76 \%$ compared to the $71 \%$ survival in those who underwent immediate cystectomy. Patients were followed up every 3-6 months, and non-invasive recurrences were treated with TURBT and, in some cases, intravesical BCG therapy. The drawback of radical TURBT is that even with regular surveillance, there is an $8-16 \%$ risk that retained, or recurrent tumor can eventually progress and cause death [54].

\section{Radiotherapy}

Radiotherapy (RT) has emerged as one of the bladder preserving modalities, and is delivered in conjunction with maximal TURBT and chemotherapy, known as combined modality therapy (CMT). The rationale behind combining chemotherapy with radiation is to address the micrometastasis that may have already developed and to prevent tumor cells from repopulating during radiation cycles $[48,55]$. CMT generally utilizes a single agent chemotherapy such as cisplatin administered along with radiation to increase radiosensitivity of the tumor. The radiation is delivered over 6 weeks following maximal local tumor ablation. Multiple prospective trials have evaluated various CMT protocols. A recently published pooled analysis demonstrated that, for patients with similar stages of MIBC, CMT has long-term disease-specific survival comparable to immediate radical cystectomy. In this study, $69 \%$ of the patients demonstrated a complete response to CMT, with 5 and 10-year disease-specific survival rates of 71 and $65 \%$, respectively. CMT can prove to be a valuable alterative for patients who are not candidates for cystectomy or those who are determined to keep their native bladders. [56] Optimal response to CMT is achieved in well-selected patients with a single tumor $<3 \mathrm{~cm}$, without hydronephrosis, and in the absence of CIS.

Radiotherapy is controversial in the adjuvant setting. A few studies from the 1980s demonstrated the high incidence of rectal toxicity and small bowel obstruction associated with adjuvant radiotherapy [57]. Better dosing strategies are now being explored to reassess adjuvant radiation after cystectomy [58].

A study by the Danish vesical cancer group compared outcomes between preoperative radiation followed by cystectomy and radical irradiation followed by salvage cystectomy in case of residual tumor. The results of their study showed higher rate of survival in the patients who underwent radiation followed by cystectomy compared to radical irradiation with salvage cystectomy in case of the presence of residual tumor and demonstrated that radiation alone is not very effective in treating MIBC [59].

\section{Adjuvant Chemotherapy}

Adjuvant chemotherapy can be given to patients at high risk for developing tumor recurrence after cystectomy, i.e., T3-T4 $\mathrm{N} 0 / \mathrm{N}+$ disease. It provides the option to single outpatients at high risk for surgical failure and only treat those patients with chemotherapy. The major disadvantage is that the patient undergoes a cystectomy nonetheless, and there is a delay in addressing occult metastasis. Absence of tumor recurrence is the only way to evaluate response to adjuvant chemotherapy [39]. Cisplatin-based combination chemotherapy has been shown to have a fair response rate [60]. Many adjuvant chemotherapy trials have been suspended prematurely, leading to dearth of data to make definitive recommendations.

The EORTC conducted a randomized phase 3 trial on patients with T3-T4 and patients with node positive MIBC, who underwent radical cystectomy and PLND. Patients were randomized to immediate treatment and deferred treatment groups. The study found no significant improvement in overall survival in the immediate treatment group compared to the treatment-deferred group [61]. This suggests that the timing of adjuvant or early salvage chemotherapy following cystectomy does not impact survival. Despite lack of level 1 evidence supporting the use of adjuvant chemotherapy, it is still widely administered [62].

\section{Urinary Diversions}

After the cystectomy has been performed, the next step is to determine the best possible way to divert urine. Urinary diversions can either be non-continent (ileal conduit) or continent (cutaneous reservoir, orthotopic neobladder). There is no consensus on the best surgical option, and in most cases, the diversion is adapted to the patient's needs and the surgeon's expertise [63]. 
The ileal conduit is a form of non-continent diversion using a segment of ileum, which opens externally and drains continuously into a urostomy bag. The ileal conduit has a shorter operative time, and it can be performed in patients with poor baseline renal function. It has the disadvantage of being cosmetically unappealing, and is at higher risk of upper tract infection and deterioration. Since urine continuously drains out into the urostomy, there is a lesser chance of developing acidosis [64].

Continent diversions can either be cutaneous diversion or an orthotopic neobladder. The most popular cutaneous diversion is the Indiana pouch. The pouch is constructed using a tubularized segment of the distal ileum that serves as the channel to a detubularized pouch made of the cecum and proximal right colon. The Indiana pouch uses the ileocecal valve to help increase the resistance to the outflow of urine. The patient has to self-catheterize to drain urine. The disadvantage is that the patient has to drain the urine frequently and should also possess a certain amount of dexterity to do the same. Long-term complications include stone formation and acidosis [64].

A neobladder can be constructed using segments of the ileum, colon, or sigmoid colon. The terminal ileum has the capability to store urine at low pressure and can store large volumes. The segment of bowel is configured into a " $U$ " or "W" shape and detubularized to make a spherical reservoir, and it is anastomosed to the patient's native urethra [65]. The frequently used configurations are the Studer (U) neobladder or the Hautmann (W) neobladder. Ureteric anastomoses are often performed in a refluxing manner to the proximal portions of the neobladder, which are left nondetubularized. Neobladders are associated with the risk of urinary incontinence, that is, especially worse at night and urinary retention, which is particularly more common in women. The compliance and capacity of the neobladder increase overtime. So it usually takes 6-12 months postoperatively to achieve nighttime urinary control [66].

Complications common to all types of diversions include urinary tract infections (UTI), deterioration of renal function, recurrent urolithiasis (struvite stones), metabolic acidosis, stomal stenosis, and stomal herniation. The overall long-term complication rate of urinary diversions approaches $60 \%$, with stomal complication and UTIs being the most common [67].

Orthotopic neobladders are perceived to have better outcomes compared to cutaneous diversions (continent and non-continent), but surveys have shown no significant difference in overall quality of life [68].

\section{Conclusions}

Management of invasive bladder cancer is challenging and evolving. For patients with invasion into the lamina propria, an initial trial of intravesical therapy after a repeat TURBT would be customary. For patients with MIBC, particularly those with high-risk features such as hydronephrosis or lymphovascular invasion, neoadjuvant chemotherapy followed by cystectomy would be the best option. Chemotherapy followed by cystectomy would benefit patients with regionally extensive disease without distant metastasis, particularly if they respond to initial chemotherapy. For those with MIBC but no high-risk features, proceeding straight to radical cystectomy may be more appropriate. Adjuvant or salvage chemotherapy should be considered in patients who are found to have pathological T3b or N+ disease following cystectomy, when it was not suspected preoperatively. Urinary diversion is a major aspect of management of MIBC post cystectomy. There is no consensus on the best procedure for diversion. The current practice is to tailor the diversion according to the patient's general health and the surgeon's expertise. Bladder cancer is a very morbid disease. Hence, it is important to clarify what is to be expected with each treatment modality and to adequately prepare the patient.

\section{References}

1. Ferlay J, Soerjomataram I, Ervik M, Dikshit R, Eser S, Mathers C, Rebelo M, Parkin DM, Forman D, Bray F, GLOBOCAN 2012 v1.0 $\mathrm{CI}$ and MWICN 11. Cancer incidence and mortality rate.

2. Howlader N, Noone AM, Krapcho M, Garshell J, Miller D, Altekruse SF, Kosary CL, Yu M, Ruhl J, Tatalovich Z, Mariotto A, Lewis DR, Chen HS, Feuer EJ, Cronin KA (eds). SEER Cancer Statistics Review, 1975-2012, National Cancer Institute. Bethesda M. SEER cancer statistics. http://seer.cancer.gov/csr/1975_2012/.

3. Siegel R, Ma J, Zou Z, Jemal A (2014) Cancer Statistics, 2014 64(1):9-29. doi:10.3322/caac.21208

4. Kaufman DS, Shipley WU, Feldman AS (2009) Bladder cancer. Lancet 374(9685):239-249. doi:10.1016/S0140-6736(09)60491-8

5. Konety B R. CSS. Management of Bladder Cancer. Springer; 2015.

6. Van den Bosch S, Witjes AJ (2011) Long-term cancer-specific survival in patients with high-risk, non-muscle-invasive bladder cancer and tumour progression: a systematic review. Eur Urol 60(3):493500. doi:10.1016/j.eururo.2011.05.045

7. Daneshmand S (2013) Determining the role of cystectomy for highgrade T1 urothelial carcinoma. Urol Clin North Am. 40(2):233247. doi:10.1016/j.ucl.2013.01.003

8. Dutta SC, Smith JA, Shappell SB, Coffey CS, Chang SS, Cookson MS (2001) Clinical under staging of high risk nonmuscle invasive urothelial carcinoma treated with radical cystectomy. J Urol 166(2): 490-493. doi:10.1016/S0022-5347(05)65969-1

9. Suárez C, Puente J, Gallardo E et al (2015) New advances in genitourinary cancer: evidence gathered in 2014. Cancer Metastasis Rev. doi:10.1007/s10555-015-9577-x

10. Gakis G, Efstathiou J, Lerner SP et al (2013) ICUD-EAU International Consultation on Bladder Cancer 2012: radical cystectomy and bladder preservation for muscle-invasive urothelial carcinoma of the bladder. Eur Urol 63(1):45-57. doi:10.1016/j. eururo.2012.08.009

11. Stimson CJ, Cookson MS, Barocas DA et al (2010) Preoperative hydronephrosis predicts extravesical and node positive disease in patients undergoing cystectomy for bladder cancer. J Urol 183(5): 1732-1737. doi:10.1016/j.juro.2010.01.028

12. Sun M, Trinh Q-D (2015) Diagnosis and staging of bladder cancer. Hematol Oncol Clin North Am 29(2):205-218. doi:10.1016/j. hoc.2014.10.013 
13. Cha EK, Tirsar LA, Schwentner C et al (2012) Accurate risk assessment of patients with asymptomatic hematuria for the presence of bladder cancer. World J Urol 30(6):847-852. doi:10.1007/s00345-012-0979-x

14. Jimbo M (2010) Evaluation and management of hematuria. Prim Care-Clin Off Pract 37(3):461-472. doi:10.1016/j.pop. 2010.04.006

15. Karakiewicz PI, Benayoun S, Zippe C et al (2006) Institutional variability in the accuracy of urinary cytology for predicting recurrence of transitional cell carcinoma of the bladder. BJU Int 97(5): 997-1001. doi:10.1111/j.1464-410X.2006.06036.X

16. Atsu N, Ekici S, Oge O, Ergen A, Hascelik G, Ozen H (2002) Falsepositive results of the NMP22 test due to hematuria. J Urol 167(2): 555-558. doi:10.1016/S0022-5347(01)69084-0

17. Sarosdy MF (1995) deVere White RW, Soloway MS, et al. Results of a multicenter trial using the BTA test to monitor for and diagnose recurrent bladder cancer. J Urol 154(2 Pt 1):379-383 . doi:10.1097 /00005392-199508000-00013discussion 383-384

18. Hajdinjak T (2008) UroVysion FISH test for detecting urothelial cancers: meta-analysis of diagnostic accuracy and comparison with urinary cytology testing. Urol Oncol Semin Orig Investig. 26(6): 646-651. doi:10.1016/j.urolonc.2007.06.002

19. Minggen Y, Zhouda Z, Zhiming Z, Xiaokun Z, Zhenqiang X, Haili L (2014) ImmunoCyt (TM) and cytology for diagnosis of bladder carcinoma: a meta-analysis. Chin Med J 127(4):758-764. doi:10.3760/cma.j.issn.0366-6999.20131900

20. Yang LPH (2014) Hexaminolevulinate blue light cystoscopy: a review of its use in the diagnosis of bladder cancer. Mol Diagn Ther 18(1):105-116. doi:10.1007/s40291-013-0068-x

21. Winters JC, Dmochowski RR, Goldman HB, et al. American Urological Association ( AUA ) Guideline American Urological Association Adult Urodynamics. 2012:1-30.

22. Fradet Y, Grossman HB, Gomella L et al (2007) A comparison of hexaminolevulinate fluorescence cystoscopy and white light cystoscopy for the detection of carcinoma in situ in patients with bladder cancer: a phase III, multicenter study. J Urol 178(1):68-73. doi:10.1016/j.juro.2007.03.028

23. Witjes JA, Redorta JP, Jacqmin D et al (2010) Hexaminolevulinateguided fluorescence cystoscopy in the diagnosis and follow-up of patients with non-muscle-invasive bladder cancer: review of the evidence and recommendations. Eur Urol 57(4):607-614. doi:10.1016/j.eururo.2010.01.025

24. Lerner SP, Goh AC, Tresser NJ, Shen SS (2008) Optical coherence tomography as an adjunct to white light cystoscopy for intravesical real-time imaging and staging of bladder cancer. Urology 72(1): 133-137. doi:10.1016/j.urology.2008.02.002

25. Chen G, Wang B, Li H, Ma X, Shi T, Zhang X (2013) Applying narrow-band imaging in complement with white-light imaging cystoscopy in the detection of urothelial carcinoma of the bladder. Urol Oncol Semin Orig Investig 31(4):475-479. doi:10.1016/j. urolonc.2011.02.009

26. Herr HW, Donat SM (2008) A comparison of white-light cystoscopy and narrow-band imaging cystoscopy to detect bladder tumour recurrences. BJU Int 102(9):1111-1114. doi:10.1111/j.1464-410 X.2008.07846.x

27. MacVicar AD (2000) Bladder cancer staging. BJU Int 86(Suppl 1): $111-122$

28. Cowan NC, Crew JP (2010) Imaging bladder cancer. Curr Opin Urol 20(5):409-413. doi:10.1097/MOU.0b013e32833 cbcb9

29. Kim JK, Park S-Y, Ahn HJ, Kim CS, Cho K-S (2004) Bladder cancer: analysis of multi-detector row helical CT enhancement pattern and accuracy in tumor detection and perivesical staging. Radiology 231(3):725-731. doi:10.1148/radiol.2313021253

30. Setty BN, Holalkere NS, Sahani DV, Uppot RN, Harisinghani M, Blake MA (2007) State-of-the-art cross-sectional imaging in bladder cancer. Curr Probl Diagn Radiol 36(2):83-96. doi:10.1067/j.cpradiol.2006.12.005

31. Liedberg F, Bendahl P-O, Davidsson T et al (2012) Preoperative staging of locally advanced bladder cancer before radical cystectomy using 3 tesla magnetic resonance imaging with a standardized protocol. Scand J Urol Nephrol 2012:1-5. doi:10.3109 100365599.2012.721394

32. Paik ML, Scolieri MJ, Brown SL, Spirnak JP, Resnick MI (2000) Limitations of computerized tomography in staging invasive bladder cancer before radical cystectomy. J Urol 163(6):1693-1696. doi:10.1016/S0022-5347(05)67522-2

33. Aljabery F, Lindblom G, Skoog S et al (2015) PET/CT versus conventional $\mathrm{CT}$ for detection of lymph node metastases in patients with locally advanced bladder cancer. BMC Urol 15(1):87. doi:10.1186/s12894-015-0080-Z

34. Zheng J (2012) Energy metabolism of cancer: glycolysis versus oxidative phosphorylation (review). Oncol Lett 4(6):1151-1157. doi:10.3892/ol.2012.928

35. Williams RD (2006) Combined metabolic/anatomical imaging in urologic oncology. J Urol 176(3):863. doi:10.1016/j. juro.2006.06.102

36. Bouchelouche K, Choyke PL (2015) PET/computed tomography in renal, bladder, and testicular cancer. PET Clin 10(3):361-374. doi:10.1016/j.cpet.2015.03.002

37. Apolo AB, Riches J, Schöder H et al (2010) Clinical value of fluorine-18 2-fluoro-2-deoxy-D-glucose positron emission tomography/computed tomography in bladder cancer. J Clin Oncol 28(25):3973-3978. doi:10.1200/JCO.2010.28.7052

38. Zaid HB, Patel SG, Stimson CJ et al (2014) Trends in the utilization of neoadjuvant chemotherapy in muscle-invasive bladder cancer: results from the national cancer database. Urology 83(1):75-80. doi:10.1016/j.urology.2013.07.072

39. Calabrò F, Sternberg CN (2009) Neoadjuvant and adjuvant chemotherapy in muscle-invasive bladder cancer. Eur Urol 55(2):348358. doi:10.1016/j.eururo.2008.10.016

40. Teramukai S, Nishiyama H, Matsui Y, Ogawa O, Fukushima M (2006) Evaluation for surrogacy of end points by using data from observational studies: tumor downstaging for evaluating neoadjuvant chemotherapy in invasive bladder cancer. Clin Cancer Res 12(1):139-143. doi:10.1158/1078-0432.CCR-05-1598

41. Balar AV, Milowsky MI (2015) Neoadjuvant therapy in muscleinvasive bladder cancer. Urol Clin North Am 42(2):217-224. doi:10.1016/j.ucl.2015.02.004

42. Neoadjuvant cisplatin,methotrexate, and vinblastine chemotherapy for muscle-invasive bladder cancer: a randomised controlled trial. Lancet. 1999; 354(9178):533-540. doi:10.1016 /S0140-6736(99)02292-8.

43. Grossman HB, Natale RB, Tangen CM et al (2003) Neoadjuvant chemotherapy plus cystectomy compared with cystectomy alone for locally advanced bladder cancer. N Engl J Med 349(9):859 866. doi:10.1056/NEJMoa022148

44. Roberts JT, von der Maase H, Sengeløv L et al (2006) Longterm survival results of a randomized trial comparing gemcitabine/cisplatin and methotrexate/vinblastine/doxorubicin/cisplatin in patients with locally advanced and metastatic bladder cancer. Ann Oncol 17(Suppl 5(Supplement 5)):v118 v122. doi:10.1093/annonc/mdj965

45. Rose TL, Deal AM, Basch E et al (2015) Neoadjuvant chemotherapy administration and time to cystectomy for muscle-invasive bladder cancer: an evaluation of transitions between academic and community settings. Urol Oncol. doi:10.1016/j. urolonc.2015.05.028

46. Culp SH, Dickstein RJ, Grossman HB et al (2014) Refining patient selection for neoadjuvant chemotherapy before radical cystectomy. J Urol 191(1):40-47. doi:10.1016/j.juro.2013.07.061 
47. Springer C, Mohammed N, Alba S et al (2014) Laparoscopic radical cystectomy with extracorporeal ileal neobladder for muscle-invasive urothelial carcinoma of the bladder: technique and short-term outcomes. World J Urol 32(2):407-412. doi:10.1007/s00345-013-1122-3

48. Stein JP, Lieskovsky G, Cote R et al (2001) Radical cystectomy in the treatment of invasive bladder cancer: long-term results in 1,054 patients. J Clin Oncol 19(3):666-675

49. Fonseka T, Ahmed K, Froghi S, Khan SA. Comparing robotic, laparoscopic and open cystectomy: a systematic review and metaanalysis. 2015; (1):41-48.

50. Guru KA, Perlmutter AE, Butt ZM, et al. The learning curve for robot-assisted radical cystectomy 2009; 13(4):509-514. doi:10.4293/108680809X12589998404128.

51. Stein JP (2006) Lymphadenectomy in bladder cancer: how high is "high enough"? Urol Oncol 24(4):349-355. doi:10.1016/j. urolonc.2005.07.013

52. Herr HW. An impact on bladder cancer outcomes after radical cystectomy. Urology. 4295(02):105-108.

53. Leissner J, Ghoneim MA, Abol-Enein H et al (2004) Extended radical lymphadenectomy in patients with urothelial bladder cancer: results of a prospective multicenter study. J Urol 171(1):139-144. doi:10.1097/01.ju.0000102302.26806.fb

54. Herr BHW (2015) Transurethral resection of muscle-invasive bladder cancer. 10-Year Outcome 19(1):89-93

55. Hautmann RE, de Petriconi RC, Pfeiffer C, Volkmer BG (2012) Radical cystectomy for urothelial carcinoma of the bladder without neoadjuvant or adjuvant therapy: long-term results in 1100 patients. Eur Urol 61(5):1039-1047. doi:10.1016/j.eururo.2012.02.028

56. Mak RH, Hunt D, Shipley WU et al (2014) Long-term outcomes in patients with muscle-invasive bladder cancer after selective bladder-preserving combined-modality therapy: a pooled analysis of radiation therapy oncology group protocols $8802,8903,9506$, 9706, 9906, and 0233. J Clin Oncol 32(34):3801-3809. doi:10.1200/JCO.2014.57.5548

57. Kopelson Gene HJ. Postoperative radiation therapy for muscle-invading bladder carcinoma. J Clin Oncol. Volume 23(Issue 4,):pages 263-268.

58. Baumann BC, Noa K, Wileyto EP et al (2015) Adjuvant radiation therapy for bladder cancer: a dosimetric comparison of techniques. Med Dosim 1-6. doi:10.1016/j.meddos.2015.06.001
59. Sell A, Jakobsen A, Nerstrøm B, Sørensen BL, Steven KBH. Treatment of advanced bladder cancer category T2 T3 and T4a. A randomized multicenter study of preoperative irradiation and cystectomy versus radical irradiation and early salvage cystectomy for residual tumor. DAVECA protocol 8201. Danish Vesical Cancer. Scand J Urol Nephrol Suppl 1991; 138193-201.

60. Sternberg CN, Bellmunt J, Sonpavde G et al (2013) ICUDEAU International Consultation on Bladder Cancer 2012: chemotherapy for urothelial carcinoma-neoadjuvant and adjuvant settings. Eur Urol 63(1):58-66. doi:10.1016/j. eururo.2012.08.010

61. Sternberg CN, Skoneczna I, Kerst JM et al (2015) Immediate versus deferred chemotherapy after radical cystectomy in patients with pT3-pT4 or N+ M0 urothelial carcinoma of the bladder (EORTC 30994): an intergroup, open-label, randomised phase 3 trial. Lancet Oncol 16(1):76-86. doi:10.1016/S1470-2045(14)71160-X

62. David KA, Milowsky MI, Ritchey J, Carroll PR, Nanus DM (2007) Low incidence of perioperative chemotherapy for stage III bladder cancer 1998 to 2003: a report from the National Cancer Data Base. J Urol 178(2):451-454. doi:10.1016/j.juro.2007.03.101

63. Hautmann RE, Abol-Enein H, Lee CT et al (2015) Urinary diversion: how experts divert. Urology 85(1):233-238. doi:10.1016/j. urology.2014.06.075

64. Bachir BG, Kassouf W (2013) Urinary diversions. Curr Opin Support Palliat Care 7(3):249-253. doi:10.1097/SPC.0b013 e3283627903

65. Lawrentschuk N, Chang DS (2015) Orthotopic neobladder reconstruction. Urol Ann 7(1):1. doi:10.4103/0974-7796.148553

66. Hautmann RE, Abol-Enein H, Hafez K et al (2007) Urinary diversion. Urology 69(1):17-49. doi:10.1016/j.urology.2006.05.058

67. Yong SM, Dublin N, Pickard R, Cody DJ, Neal DE, N'Dow J. Urinary diversion and bladder reconstruction/replacement using intestinal segments for intractable incontinence or following cystectomy. Cochrane Database Syst Rev. 2003; (1):CD003306. doi:10.1002/14651858.CD003306.

68. Dutta SC, Chang SC, Coffey CS, Smith JA, Jack G, Cookson MS (2002) Health related quality of life assessment after radical cystectomy: comparison of ileal conduit with continent orthotopic neobladder. J Urol 168(1):164-167. doi:10.1097/00005392200207000-00037 